\title{
Evidências cientificas sobre a associação entre burnout e síndrome metabólica: revisão integrativa
}

Scientific evidence on the association between burnout and metabolic syndrome: integrative review

Evidencias cientificas sobre la asociación entre el burnout y el síndrome metabólico: revisión integradora

Magno Conceição das Merces ${ }^{1}$

Antonio Marcos Tosoli Gomes ${ }^{2}$

Julita Maria Freitas Coelho ${ }^{3}$

Maria Lúcia Silva Servo ${ }^{4}$

Sergio Correa Marques ${ }^{2}$

Argemiro D’Oliveira Júnior ${ }^{1}$

\section{Descritores}

Esgotamento profissional; Síndrome metabólica; Saúde do trabalhador; Epidemiologia

\section{Keywords}

Burnout, professional; Metabolic syndrome;

Occupational health; Epidemiology

\section{Descriptores}

Agotamiento profesional; Síndrome metabólico;

Salud laboral; Epidemiología

\section{Submetido}

25 de Março de 2019

Aceito

6 de Maio de 2019

\section{Resumo}

Objetivo: Avaliar as evidências científicas disponiveis na literatura sobre a associação entre Síndrome de Burnoute Síndrome Metabólica. Métodos: Revisão integrativa da literatura, com buscas nas bases de dados do Cumulative Index to Nursing and Allied Health Literature, National Library of Medicine National Institutes of Health, Web of Science, Scopus, Cochrane Library, Latin American and Caribbean Center on Health Sciences Information, Scientific Eletronic Library Online e Springer Link. Os artigos selecionados foram analisados de acordo com a Agency for Healthcare Research and Quality.

Resultados: A maioria (80\%) dos 5 artigos que atenderam aos critérios de seleção estava na língua inglesa e indexada nas bases de dados Web of Science e Scopus. Dentre os trabalhos, $80 \%$ tinham médicos como autores principais. 0 continente asiático (Israel, Japão e China) concentrou a maior parte de produção. Não ocorreu predominância de desenho de estudo. A área financeira correspondeu a 60\% do público pesquisado. Conclusão: As evidências disponíveis na literatura são incipientes, apenas $20 \%$ dos artigos elegiveis apresentou associação entre as síndromes estudadas e os demais, indicam associação entre Burnout e componentes da SM separadamente.

\section{Abstract}

Objective: To evaluate the scientific evidence available in the literature on the association between Burnout Syndrome and Metabolic Syndrome. Methods: Integrative literature review, searching the databases of the Cumulative Index to Nursing and Allied Health Literature, National Library of Medicine, National Institutes of Health, Web of Science, Scopus, Cochrane Library, Latin American and Caribbean Center on Health Sciences Information, Scientific Eletronic Library Online and Springer Link. The selected articles were analyzed according to the Agency for Healthcare Research and Quality.

Results: Most (80\%) of the 5 studies that met the selection criteria were in English language and indexed in the Web of Science and Scopus databases. Among the studies, $80 \%$ had physicians as the main authors. The Asian continent (Israel, Japan and China) concentrated the majority of production. There was no predominance of study design. The financial area corresponded to $60 \%$ of the study participants.

Conclusion: The available evidence in the literature is incipient, only $20 \%$ of the eligible articles showed association between the syndromes studied and the others, indicate association between Burnout and MS components separately.

\section{Resumen}

Objetivo: evaluar las evidencias científicas disponibles en la literatura sobre la asociación entre el síndrome de burnouty el síndrome metabólico. Métodos: revisión integradora de la literatura, con búsquedas en las bases de datos del Cumulative Index to Nursing and Allied Health Literature, National Library of Medicine National Institutes of Health, Web of Science, Scopus, Cochrane Library, Latin American and Caribbean Center on Health Sciences Information, Scientific Eletronic Library Online y Springer Link. Los artículos seleccionados fueron analizados de acuerdo con la Agency for Healthcare Research and Quality.

Resultados: la mayoría (80\%) de los cinco artículos que cumplieron los criterios de selección estaba en inglés e indexada en las bases de datos Web of Science y Scopus. Entre los trabajos, $80 \%$ tenía médicos como autores principales. El continente asiático (Israel, Japón y China) concentró la mayor parte de la producción. No ocurrió predominio de diseño de estudio. El área financiera correspondió al $60 \%$ del público investigado. Conclusión: las evidencias disponibles en la literatura son incipientes, solo el 20\% de los artículos elegibles presentó asociación entre los síndromes estudiados y los demás indican asociación entre burnout y componentes del SM separadamente.

\section{Autor correspondente}

Magno Conceição das Merces

https://orcid.org/0000-0003-3493-8606

E-mail: mmerces@uneb.br

\section{DOI}

http://dx.doi.org/10.1590/1982-

0194201900064

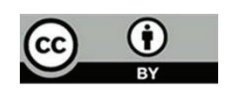

\section{Como citar:}

Merces MC, Gomes AM, Coelho JM, Servo ML, Marques SC, D'Oliveira Júnior A. Evidências científicas sobre a associação entre burnout e síndrome metabólica: revisão integrativa. Acta Paul Enferm. 2019;32(4):470-6.

\section{'Faculdade de Medicina, Universidade Federal da Bahia, Salvador, BA, Brasil.}

2Faculdade de Enfermagem, Universidade do Estado do Rio de Janeiro, Rio de Janeiro, RJ, Brasil. ${ }^{3}$ Departamento de Ciências da Vida, Universidade do Estado da Bahia, Salvador, BA, Brasil.

${ }^{4}$ Departamento de Saúde, Universidade Estadual de Feria de Santana, Feira de Santana, BA, Brasil.

Conflitos de interesse: artigo extraído da tese de doutorado "Síndrome de Burnout e Síndrome Metabólica em Profissionais de Enfermagem da Atenção Primária à Saúde", desenvolvida na Faculdade de Medicina, Universidade Federal da Bahia, Salvador, Bahia, Brasil. 


\section{Introdução}

As múltiplas metamorfoses que ocorrem no mundo do trabalho no Brasil são notáveis, principalmente pela crise ideológica, social e política inconsequente de austeridade. No contexto do trabalho, o cerne perpassa pela proletarização e subproletarização de segmentos produtivos e de serviços em uma perspectiva desenfreada e irreversível, fugindo metaforicamente da curva de Gauss. ${ }^{(1)}$

A proletarização é pautada na perda da autonomia sobre o processo de trabalho e a subproletarização, como trabalho precário, temporário, terceirizado e fragmentado. ${ }^{(1)}$ Estas condiçôes proporcionam, aos ambientes das organizações de trabalho, sofrimentos, adversidades e condiçôes aviltantes de exploração. A lógica da privatização, posta como onda senoidal suave, apresenta desfechos nefastos à saúde do trabalhador.

Frente a esse cenário complexo, advém o desgaste mental no trabalho, surgindo os Transtornos Mentais Comuns (TMC), estresse ocupacional e Síndrome de Burnout (SB). A SB, também conhecida como síndrome do esgotamento profissional, é um fenômeno iniciado pelo estresse crônico no trabalho e acomete principalmente trabalhadores que possuem contato contínuo com pessoas. A SB possui três dimensôes relacionadas e independentes, a saber: (a) exaustão emocional, que se refere à falta de energia, esgotamento físico e mental; (b) despersonalização, que dá origem à insensibilidade emocional; (c) reduzida realização profissional, geradora de insatisfação com as atividades laborais, baixa autoestima, redução da interação com os pares, sentimento de incompetência. ${ }^{(2)}$

A prevalência da $S B$ possui variação nas categorias profissionais, sendo 4,8\% a 39,3\% nos profissionais de Saúde, $54,9 \%$ a $56 \%$ em policiais, $5,7 \%$ a $15,4 \%$ em professores. ${ }^{(3-8)}$ Estas variaçôes entre as prevalências decorrem da utilização de critérios não robustos para definição da síndrome. Estudos apontam que a SB é fator de exposição para: TMC, iatrogenias, uso de substâncias psicoativas, ideação suicida e tentativa de suicídio, dor musculoesquelética, distúrbios do sono, imunidade prejudicada, adiposidade abdominal, resistência à insulina, hipercolesterolemia, diabetes tipo 2 e Síndrome Metabólica (SM).(9-19)
A SM, de acordo a National Cholesterol Education Program's Adult Treatment Panel III e a I Diretriz Brasileira de Diagnóstico e Tratamento da Síndrome Metabólica, caracteriza-se como “[...] um transtorno complexo representado por um conjunto de fatores de risco cardiovascular usualmente relacionados à deposição central de gordura e à resistência à insulina”. ${ }^{(20)}$ Para diagnóstico da SM, o indivíduo deve apresentar a combinação de pelo menos três dos cinco componentes: circunferência abdominal elevada, hipertrigliceridemia, redução do HDL colesterol, Hipertensão Arterial Sistêmica e hiperglicemia. A associação da SM com a doença cardiovascular eleva a mortalidade geral em aproximadamente 1,5 vezes e a cardiovascular em 2,5 vezes. ${ }^{(20)}$

Investigaçóes sobre a SM têm sido conduzidas em distintas populações, a exemplo da venezuelana, mexicana, norte-americana e asiática, e a sua ocorrência, embora alta, apresenta variaçóes. Pontua-se que, de 2003 a 2012, a prevalência geral da SM nos Estados Unidos foi de 33\%, com supremacia significativa em mulheres em comparação aos homens. ${ }^{(20,21)}$ Dados alusivos à predominância da SM no Brasil são escassos e não evidenciam a real ocorrência desse evento em nível populacional.

A literatura aponta fatores de exposição para a SM, como: periodontite, Acantose nigricans, doença hepática gordurosa não alcoólica, biomarcadores (adipocinas, neuropeptídeos, citocinas pró-inflamatórias, citocinas anti-inflamatórias, marcadores de status antioxidante e fatores pró-trombóticos), esquizofrenia, ansiedade, condições de trabalho, estresse ocupacional e SB. ${ }^{(22-29)}$ No entanto, muitos outros fatores ainda não foram identificados.

As transformaçóes do mundo do trabalho e as atuais condiçóes precárias e também a exposição crônica a múltiplos fatores deletérios levam ao quadro de estresse ocupacional e a exacerbação deste, à presença da SB. Por conseguinte, essas condiçóes concorrerão para a redução da resiliência biológica e, portanto, afetarão a homeostase, contribuindo para o desenvolvimento da SM. ${ }^{(16,30)}$

Assim, situações de trabalho estressantes estimulam a resposta do eixo Hipotálamo Hipófise Adrenal (HHA), levando à resistência insulínica em consequência da excessiva produção de cortisol. $\mathrm{O}$ 
aumento dos níveis de cortisol liberado pelo córtex adrenal, relacionado, por sua vez, à estimulação do hormônio adrenocorticotrófico (ACTH) liberado pela hipófise, estaria relacionado à adiposidade abdominal, pois há a mobilização de lipídeos a partir do tecido adiposo, e da glicose, a partir do glicogênio hepático, visando aumentar a quantidade de energia disponível para as situaçôes de estresse. ${ }^{(16)}$

O objetivo do estudo é avaliar as evidências científicas disponíveis na literatura sobre a associação entre Síndrome de Burnout e Síndrome Metabólica.

\section{Métodos}

Foi conduzida uma revisão integrativa da literatura, que reúne, avalia e sintetiza achados provenientes de estudos primários, fundamentados em evidências científicas disponíveis sobre determinado tema. Para sistematização dessa revisão, foram seguidas seis etapas: elaboração da pergunta norteadora; busca ou amostragem na literatura; coleta de dados; análise crítica dos estudos incluídos; discussão dos resultados; apresentação da revisão integrativa. ${ }^{(31)}$

Destaca-se que foram seguidas as recomendaçóes do checklist do Statement for Reporting Systematic Reviews and Meta-Analyses of Studie (PRISMA). Nesse sentido, utilizou-se a estratégia PICO para elaboração da pergunta norteadora. Essa estratégia representa um acrônimo para Paciente, Intervenção, Comparação e Outcomes (desfecho). ${ }^{(32)}$ Outrossim, o P se referiu aos trabalhadores expostos ao desenvolvimento de SB e SM, I ao lócus de trabalho estressante, $\mathrm{C}$ comparaçóes entre os níveis de evidências científicas e $\mathrm{O}$ possível associação entre $\mathrm{SB}$ e SM. Logo, obteve-se a questão norteadora: Quais as evidências científicas disponíveis na literatura sobre a associação entre $\mathrm{SB}$ e $\mathrm{SM}$ ?

A busca das publicaçóes foi realizada de setembro a dezembro de 2018, utilizando-se operador booleano $A N D$ e técnicas de truncamento, nos artigos publicados nas seguintes bases de dados: Cumulative Index to Nursing and Allied Health Literature (CINAHL), National Library of Medicine National Institutes of Health (PubMed), Web of Science, Scopus, Cochrane Library, Latin
American and Caribbean Center on Health Sciences Information (LILACS), Scientific Eletronic Library Online (SciElo) e Springer Link. As palavras-chave foram definidas segundo os descritores em ciências da saúde (DeCS) e o Medical Subject Headings $(\mathrm{MeSH})$, em português, espanhol e inglês, sendo: Esgotamento Profissional, Agotamiento Profesional, Burnout, Profesional; Síndrome Metabólica, Sindrome Metabólico, Metabolic Syndrome.

Consideraram-se elegíveis os artigos publicados em português, espanhol e inglês, sem limite de data, disponíveis eletronicamente nas bases supracitadas e que tratassem da associação entre Burnout e SM. Os critérios de exclusão foram: artigos que não apresentavam relação com o objeto; relatos de experiências e de casos, monografias, dissertaçóes, teses, resumos em anais de eventos, capítulos de livro. Artigos duplicados foram considerados uma única vez.

Um instrumento construído foi empregado para coleta e construçáo do banco de dados, contendo informaçôes que incluíam: identificação; instituição sede do estudo; tipo de publicação; características metodológicas do estudo; e avaliação do rigor metodológico.

Tendo em vista a análise crítica dos estudos elegíveis, adotou-se a classificação dos níveis de evidência científica da Agency for Healthcare Research and Quality (AHRQ), que abrange seis níveis: (I) evidências resultantes de metanálise e revisão sistemática; (II) evidências obtidas em ensaios clínicos com randomização; (III) evidências obtidas em ensaios clínicos sem randomização; (IV) evidências de estudos de coorte e de caso-controle; (V) evidências oriundas de revisão sistemática de estudos descritivos e qualitativos; (VI) evidências baseadas em estudo descritivo ou qualitativo.

Após leitura e análise crítica dos artigos, elaborou-se um quadro sinóptico com síntese das publicaçóes selecionadas, contendo autor/ano/revista, país onde o estudo foi conduzido, consideraçóes temáticas, tipo de estudo, síntese das conclusóes e classificação AHRQ.

\section{Resultados}

Foram encontrados 199 artigos nas bases de dados, a saber: 22,6\% CINAHL, 25,6\% PubMed, 13,1\% 
Web of Science, 21,6\% Scopus, 5,5\% Cochrane Library, 2,5\% LILACS, 3,1\% SciElo e 6,0\% Springer Link. Pautando-se nos critérios de elegibilidade e na análise detalhada das publicações, 194 artigos não atenderam aos critérios, equivalendo a: $10,9 \%$ duplicados, $88,1 \%$ não apresentavam relação com o objeto de estudo, $0,5 \%$ em outra língua e $0,5 \%$ relato de caso. Frente ao exposto, nesta revisão, foram selecionados cinco artigos, que estão sumarizados na figura 1 .

Dos cinco estudos elegíveis, $80 \%$ encontravamse em língua inglesa e $20 \%$ em espanhol, indexados em sua maioria nas bases de dados Web of Science e Scopus. Destaca-se que quatro (80\%) periódicos eram da área de saúde do trabalhador e apenas um (20\%) da área médica. Quanto aos anos de publicação, incidiram entre 2006 e 2018. Os critérios de busca não incluíram corte temporal. No tocante à formação dos autores principais de cada estudo, $80 \%$ eram médicos e $20 \%$ enfermeiros. Houve maior concentração de artigos no continente asiático - Israel, Japão e China - (60\%). Na Europa, os países que conduziram estudos foram Espanha e França, representando $40 \%$ da amostra.
Não ocorreu predominância de desenho de estudo, sendo: revisão de literatura, transversal, coorte, quase-experimental não randomizado e caso-controle, representando $20 \%$ cada. A população investigada nos estudos foi majoritariamente constituída de profissionais que desenvolviam atividades laborais na área financeira (60\%). Um único estudo foi conduzido com professores do ensino médio, não sendo identificada pesquisa com trabalhadores da saúde.

De acordo com as categorias do AHRQ, 40\% dos artigos foram classificados como nível de evidência IV (coorte e caso-controle); 20\%, nível de evidência V (revisão de literatura); 20\%, nível de evidência VI (transversal); e 20\%, nível de evidência III (quase experimental não-randomizado).

Todos os estudos que compuseram a revisão integrativa da literatura descreviam o Burnout como variável preditora para a SM. Em 20\% dos estudos, ficou evidente a associação entre as síndromes; em 60\%, a associação entre Burnout e componentes da SM separadamente. Os artigos desta revisão estão sintetizados no quadro 1 , ordenados de acordo com o ano de publicação.

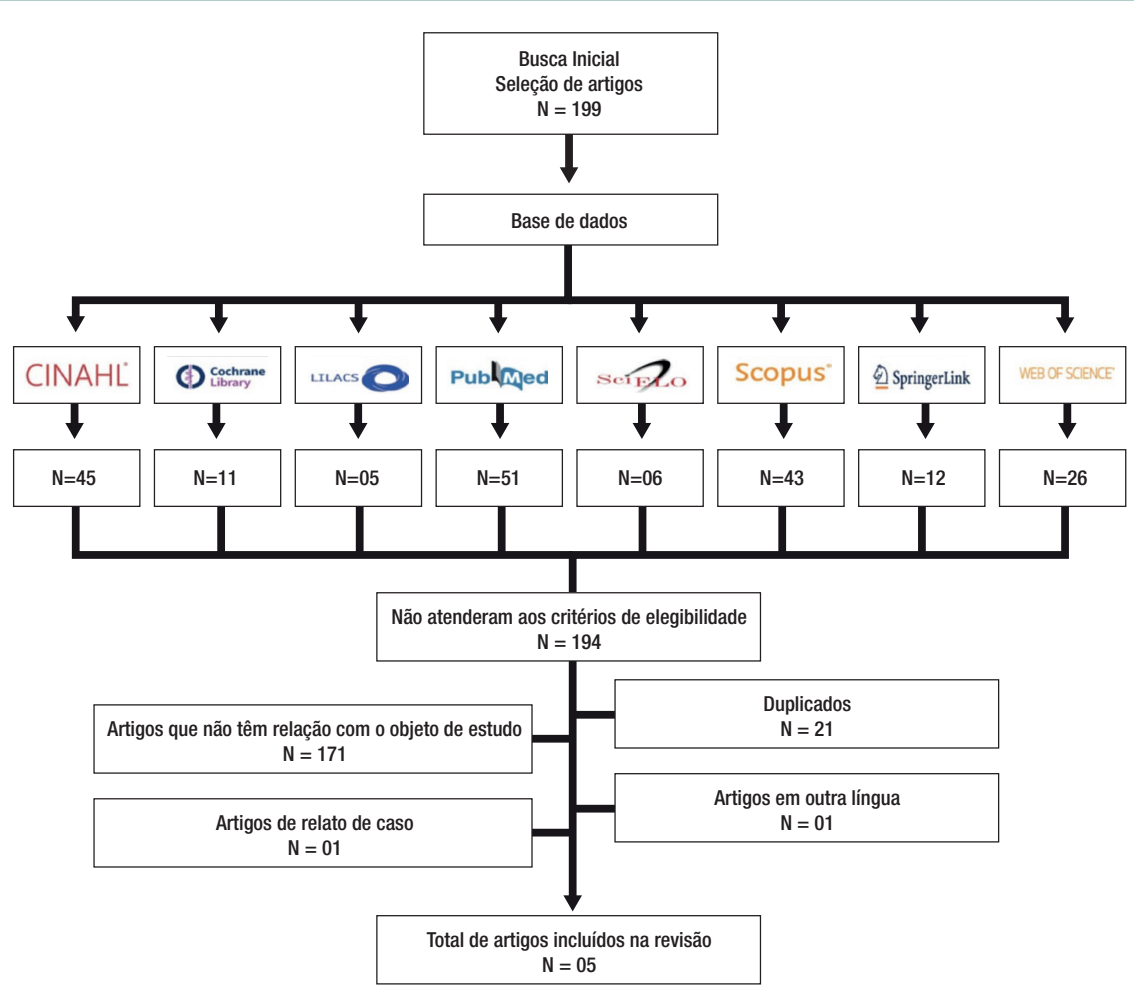

Figura 1. Fluxograma da revisão integrativa sobre evidências científicas entre Burnoute Síndrome Metabólica 
Quadro 1. Caracterização das publicações incluídas na revisão integrativa, segundo ano/autor /revista, tipo e país onde o estudo foi conduzido, nível de evidência científica, considerações temáticas, síntese das conclusões

\begin{tabular}{|c|c|c|c|}
\hline Ano de publicação/ Autor/Revista & $\begin{array}{l}\text { Tipo e país do estudo/ } \\
\text { Nível de evidência }\end{array}$ & Considerações temáticas & Síntese das conclusões \\
\hline $\begin{array}{l}2006 \\
\text { Melamed S, Shirom A, Toker S, Berliner } \\
\text { S, Shapira I(15) } \\
\text { Psychological Bulletin }\end{array}$ & $\begin{array}{l}\text { Revisão de literatura } \\
\text { Israel } \\
\text { Nível V }\end{array}$ & $\begin{array}{l}\text { Avaliam a evidência acumulada na literatura internacional } \\
\text { sobre Burnout e exaustão vital associados com o risco } \\
\text { aumentado de eventos cardiovasculares, pontuando a } \\
\text { Síndrome Metabólica. }\end{array}$ & $\begin{array}{l}0 \text { Burnout pode desencadear distúrbios do sono, depressão, } \\
\text { processos inflamatórios, alterações imunológicas, diabetes mellitus } \\
\text { Síndrome Metabólica e outros eventos cardiometabólicos. }\end{array}$ \\
\hline $\begin{array}{l}2008 \\
\text { Ranchal-Sánchez A, Vaquero-Abellán } \\
\text { M(33) } \\
\text { Medicina y Seguridad del Trabajo }\end{array}$ & \begin{tabular}{|l|} 
Transversal \\
Espanha \\
Nivel VI
\end{tabular} & $\begin{array}{l}\text { Avaliam a associação entre Burnout e dosagens } \\
\text { bioquímicas, a saber: glicose sérica e os níveis de } \\
\text { colesterol, bem como o índice de massa corporal em } \\
\text { uma amostra aleatória de } 186 \text { professores do ensino } \\
\text { secundário. }\end{array}$ & $\begin{array}{l}\text { Os achados apontam que não houve associação entre o Burnoute } \\
\text { os componentes da Síndrome Metabólica, entretanto, foi encontrado } \\
\text { que quanto maior a realização profissional, que integra uma das } \\
\text { dimensões do Burnout, menor o índice de massa corporal. }\end{array}$ \\
\hline $\begin{array}{l}2009 \\
\text { Kitaoka-Higashiguchi K, Morikawa Y, } \\
\text { Miura K(34) } \\
\text { Journal of Occupational Health }\end{array}$ & \begin{tabular}{|l} 
Coorte \\
Japão \\
Nivel IV
\end{tabular} & \begin{tabular}{|l} 
Investigam o Burnout e fatores de risco para doença \\
aterosclerótica, destacando a Síndrome Metabólica entre \\
442 gerentes de nível médio de uma fábrica.
\end{tabular} & \begin{tabular}{|l|} 
Mudanças na circunferência de cintura, peso corporal e índice de \\
massa corpórea foram significativamente maiores nos gerentes com \\
Burnout. Apesar de descrever a plausibilidade biológica que aponta \\
a associacãa entre Burnout e Síndrome Metabólica, não foram \\
encontradas significâncias estatísticas no caminho descrito.
\end{tabular} \\
\hline $\begin{array}{l}2013 \\
\text { Tsai HH, Yeh CY, Su CT, Chen CJ, Peng } \\
\text { SM, Chen RY (19) } \\
\text { Industrial Health }\end{array}$ & $\begin{array}{l}\text { Quase-experimental } \\
\text { não randomizado } \\
\text { China } \\
\text { Nivel III }\end{array}$ & $\begin{array}{l}\text { Estimam que a atividade física seja um fator de } \\
\text { intervenção que afeta a relação entre o Burnoute } \\
\text { componentes da Síndrome Metabólica entre } 109 \\
\text { consultores financeiros e administradores de um banco } \\
\text { de seguros. }\end{array}$ & $\begin{array}{l}\text { Um programa de exercícios bem projetado estabelece melhor } \\
\text { comportamento de saúde no local de trabalho, aliviando Burnout } \\
\text { e componentes da Síndrome Metabólica. Existe associação entre } \\
\text { Burnout e componentes da Síndrome Metabólica. }\end{array}$ \\
\hline $\begin{array}{l}2018 \\
\text { Metlaine A, Sauvet F, Gomez-Merino D, } \\
\text { Boucher T, Elbaz M, Delafosse JY(17) } \\
\text { PloS ONE }\end{array}$ & $\begin{array}{l}\text { Caso-controle } \\
\text { França } \\
\text { Nível IV }\end{array}$ & \begin{tabular}{|l|} 
Investigam a exposição crônica ao estresse (Burnout), \\
qualidade do sono e repercussão biológica específica, em \\
particular respostas metabólicas e inflamatórias entre 140 \\
funcionários de uma empresa financeira.
\end{tabular} & $\begin{array}{l}\text { Os participantes com Burnout apresentaram maiores níveis de } \\
\text { HbA1c, glicemia, } P C R \text {, os níveis mais baixos de vitamina } D \text {, aumento } \\
\text { do número de leucócitos, neutrófilos e monócitos }(P<0,001 \text { para } \\
\text { todos) e maior colesterol total }(P=0,01) \text {. Não houve associação entre } \\
\text { Burnoute Síndrome Metabólica. }\end{array}$ \\
\hline
\end{tabular}

\section{Discussão}

Este é o primeiro estudo brasileiro a investigar as evidências científicas sobre a associação entre SB e SM. Os resultados da presente revisão integrativa contribuem com o reconhecimento da emergência em pesquisas e intervenções no cenário laboral, destacando o burnout e seus desfechos como condiçóes deletérias à saúde do trabalhador.

No contexto do Brasil, a história que envolve os direitos dos trabalhadores é abalizada por lutas e sangue derramado isento de qualquer nódoa moral. Somente no ano de 2012 foi promulgada a Política Nacional de Saúde do Trabalhador e da Trabalhadora, entretanto os dados registrados no Anuário da Previdência Social do Brasil, publicitado pelo Instituto Nacional do Seguro Social (INSS), vêm, há mais de uma década, divulgando o quantitativo de auxílios-doença concedidos, tendo os transtornos mentais e comportamentais, incluindo o Burnout, entre as principais causas de afastamento.

Nas bases de dados foram encontrados diversos estudos descritivos que apontam a frequência da SB. ${ }^{(3,8)}$ A sua magnitude, transcendência, gravidade e vulnerabilidade são claras na literatura.

A maioria dos artigos (80\%) apresenta médico como primeiro autor, todavia o objeto estudado deve ser conduzido por áreas multiprofissionais da saúde, uma vez que o cuidado ao trabalhador que apresenta a SM, tendo a SB como variável independente, necessitará de um acompanhamento multiprofissional. Além disso, a produção científica é um importante balizador no aprimoramento das açóes de cuidado em saúde. ${ }^{(2)}$

O maior número de artigos concentrou-se no continente asiático, certamente pelo investimento de países como Japão e China em Ciência e Tecnologia (C\&T). ${ }^{(35)}$ Observa-se que, no Brasil, não foram encontrados registros. Este fato justifica-se pela falta de investimento em C\&T, e o $A d$ aeternum slogan: "A pesquisa no Brasil encontra-se em consolidação".

Não foram identificadas pesquisas com trabalhadores da saúde. Ressalta-se a importância de estudos que avaliem a associação e a causalidade entre SB e SM, pois se trata também de um grupo de risco que vivencia ambientes de trabalho insalubres, com cargas horárias descomunais e resposta alostática excessiva ou ineficaz. ${ }^{(28,33,34)}$

Dos estudos identificados nesta revisão, segundo as categorias do AHRQ, a maioria é classificada como nível de evidência IV (coorte e caso-controle), o que resulta em nível de evidência mediano. Em relação à associação entre as síndromes, mais da me- 
tade indica associação entre Burnout e componentes da SM separadamente. De todos os artigos elegíveis, nenhum teve como escopo principal a associação entre as síndromes. Estes apresentavam-se como objetivos secundários ou terciários, o que pode ser apontado como limitação desta revisão integrativa.

Acredita-se na relevância em conduzir estudos epidemiológicos com análises robustas e ensaios clínicos para melhor conhecer, comparar e avaliar os efeitos da Síndrome de Burnout.

\section{Conclusão}

Conclui-se que as evidências disponíveis na literatura são incipientes, apenas $20 \%$ dos artigos elegíveis apresentou associação entre as síndromes estudadas e os demais, indicam associação entre Burnout e componentes da SM separadamente. Os resultados desta revisão sugerem que estudos futuros priorizem profissionais de saúde como participantes, uma vez que possuem demasiada exposição ao estresse laboral e à Síndrome de Burnout, e apresentem desenhos mais robustos, que expliquem melhor o caminho da associação/causalidade entre as síndromes aqui discutidas.

\section{Agradecimentos}

Apoio financeiro do Conselho Nacional de Desenvolvimento Científico e Tecnológico (CNPq), Brasil, Edital Universal - Processo número 408390/2016-6.

\section{Referências}

1. Ceolin GF. Capital crisis, work precariousness and impacts on Social Service. Serv Soc Soc. 2014 Apr;(118):239-64.

2. Merces MC, Cordeiro TM, Santana Al, Lua I, Silva DS, Alves MS, et al. Burnout syndrome in nursing workers of the primary health care. Rev Baiana Enferm. 2016;30(3):1-9.

3. Zanatta $A B$, Lucca SR. Prevalence of burnout syndrome in health professionals of an onco-hematological pediatric hospital. Rev Esc Enferm USP. 2015;49(2):253-60.

4. Navarro-González D, Ayechu-Díaz A, Huarte-Labiano I. [Prevalence of burnout syndrome and its associated factors in Primary Care staff]. Semergen. 2015;41(4):191-8. Spanish.
5. Aranda Beltrán C, Pando Moreno M, Salazar Estrada JG, Torres López TM, Aldrete Rodríguez MG. Social support, burnout syndrome and occupational exhaustion among Mexican traffic police agents. Span J Psychol. 2009;12(2):585-92.

6. Guimarães LA, Mayer VM, Bueno HP, Minari MR, Martins L. Burnout syndrome and quality of life in the military police and civilian. Rev Sul Am Psicol. 2014;2(1):98-122.

7. Ribeiro LC, Barbosa LA, Soares AS. Evaluation prevalence of burnout among public school teachers and their relationship to sociodemographic. Rev Enferm Centro-Oeste Min. 2015;5(3):1741-51.

8. Santos AA, Nascimento Sobrinho CL. Prevalence of systematic review burnout in teachers of elementary and secondary education. Rev Baiana Saúde Pública. 2011;35(2):299-19.

9. Silveira SL, Câmara SG, Amazarray MR. Burnout predictors in health professionals of primary care of Porto Alegre/RS, Brazil. Cad Saúde Coletiva. 2014;22(4):386-92.

10. Hall LH, Johnson J, Watt I, Tsipa A, O'Connor DB. Healthcare staff wellbeing, burnout, and patient safety: a systematic review. PLoS One. 2016;11(7):e0159015.

11. Morelli SG, Sapede M, Silva AT. Burnout among primary care physicians: a systematic review. Rev Bras Med Fam Comunidade. 2015;10(34):19.

12. Samuelsson M, Gustavsson JP, Petterson IL, Arnetz B, Asberg M. Suicidal feelings and work environment in psychiatric nursing personnel. Soc Psychiatry Psychiatr Epidemiol. 1997 0ct;32(7):391-7.

13. Langballe EM, Innstrand ST, Hagtvet KA, Falkum E, Gjerløw Aasland 0 . The relationship between burnout and musculoskeletal pain in seven Norwegian occupational groups. Work. 2009;32(2):179-88.

14. Pagnin D, de Queiroz V, Carvalho YT, Dutra AS, Amaral MB, Queiroz TT. The relation between burnout and sleep disorders in medical students. Acad Psychiatry. 2014;38(4):438-44.

15. Melamed S, Shirom A, Toker S, Berliner S, Shapira I. Burnout and risk of cardiovascular disease: evidence, possible causal paths, and promising research directions. Psychol Bull. 2006;132(3):327-53.

16. Merces MC, Silva DD, Lua I, Oliveira DS, Souza MC. Burnout syndrome and abdominal adiposity among Primary Health Care nursing professionals. Psicol Reflex Crit. 2016;29(44):2-8.

17. Metlaine A, Sauvet F, Gomez-Merino D, Boucher T, Elbaz M, Delafosse JY, et al. Sleep and biological parameters in professional burnout: A psychophysiological characterization. PLoS One. 2018;13(1):e0190607.

18. Melamed S, Shirom A, Toker S, Shapira I. Burnout and risk of type 2 diabetes: a prospective study of apparently healthy employed persons. Psychosom Med. 2006;68(6):863-9.

19. Tsai HH, Yeh CY, Su CT, Chen CJ, Peng SM, Chen RY. The effects of exercise program on burnout and metabolic syndrome components in banking and insurance workers. Ind Health. 2013;51(3):336-46.

20. Sociedade Brasileira de Hipertensão. I Brazilian Guideline for Diagnosis and Treatment of Metabolic Syndrome. Arq Bras Cardiol. 2005;84 (Supl 1):3-28.

21. Aguilar M, Bhuket T, Torres S, Liu B, Wong RJ. Prevalence of the metabolic syndrome in the United States, 2003-2012. JAMA. 2015;313(19):1973-4.

22. Gomes-Filho IS, das Mercês MC, de Santana Passos-Soares J, Seixas da Cruz S, Teixeira Ladeia AM, Trindade SC, et al. Severity of periodontitis and metabolic syndrome: Is there an Association? J Periodontol. 2016;87(4):357-66. 
23. Carneiro AS, Bordallo AP, Carvalho CN, Medeiros CB, Monteiro CB, Gilban DL. Association of metabolic syndrome with acanthosis nigricans in prepubertal children. Rev Ped SOPERJ. 2016;16(3):8-14.

24. Souza MR, Diniz MF, Medeiros-Filho JE, Araújo MS. Metabolic syndrome and risk factors for non-alcoholic fatty liver disease. Arq Gastroenterol. 2012;49(1):89-96.

25. Srikanthan K, Feyh A, Visweshwar H, Shapiro JI, Sodhi K. Systematic review of metabolic syndrome biomarkers: a panel for early detection, management, and risk stratification in the West Virginian Population. Int J Med Sci. 2016;13(1):25-38.

26. Malhotra N, Grover S, Chakrabarti S, Kulhara P. Metabolic syndrome in schizophrenia. Indian J Psychol Med. 2013;35(3):227-40.

27. Carroll D, Phillips AC, Thomas GN, Gale CR, Deary I, Batty GD. Generalized anxiety disorder is associated with metabolic syndrome in the Vietnam experience study. Biol Psychiatry. 2009;66(1):91-3.

28. Ribeiro RP, Marziale MH, Martins JT, Ribeiro PH, Robazzi ML, Dalmas JC. Prevalence of metabolic syndrome among nursing personnel and its association with occupational stress, anxiety and depression. Rev Lat Am Enfermagem. 2015;23(3):435-40.
29. Garbarino S, Magnavita N. Work stress and metabolic syndrome in police officers. A Prospective Study. PLoS One. 2015;10(12):e0144318.

30. Chandola T, Brunner E, Marmot M. Chronic stress at work and the metabolic syndrome: prospective study. BMJ. 2006;332(7540):521-5.

31. Ganong LH. Integrative reviews of nursing research. Res Nurs Health. 1987;10(1):1-11.

32. da Costa Santos CM, de Mattos Pimenta CA, Nobre MR. The PICO strategy for the research question construction and evidence search. Rev Lat Am Enfermagem. 2007;15(3):508-11.

33. Ranchal-Sánchez A, Vaquero-Abellán M. Protocol for the monitoring of the health of teachers with attention to the occupational disease. Med Segur Trab. 2008 ;54(211):47-60.

34. Kitaoka-Higashiguchi K, Morikawa Y, Miura K, Ishizaki M, Kido T, Naruse $Y$, et al. Burnout and risk factors for arteriosclerotic disease: follow-up study. J Occup Health. 2009;51(2):123-31.

35. Guimarães JÁ. Medical and biomedical research in Brazil: a comparison of Brazilian and international scientific performance. Ciênc Saúde Coletiva. 2004;9(2):303-27. 